\title{
Flow characteristics of hypersonic inlets with different cowl-lip blunting methods
}

\author{
LU HongBo, YUE LianJie* \& CHANG XinYu \\ State Key Laboratory of High Temperature Gas Dynamics, Institute of Mechanics, Chinese Academy of Sciences, Beijing 100190, China
}

Received March 9, 2013; accepted April 26, 2013; published online February 17, 2014

\begin{abstract}
Under hypersonic flight conditions, the sharp cowl-lip leading edges have to be blunted because of the severe aerodynamic heating. This paper proposes four cowl-lip blunting methods and studies the corresponding flow characteristics and performances of the generic hypersonic inlets by numerical simulation under the design conditions of a flight Mach number of 6 and an altitude of $26 \mathrm{~km}$. The results show that the local shock interference patterns in the vicinity of the blunted cowl-lips have a substantial influence on the flow characteristics of the hypersonic inlets even though the blunting radius is very small, which contribute to a pronounced degradation of the inlet performance. The Equal Length blunting Manner (ELM) is the most optimal in that a nearly even reflection of the ramp shock produces an approximately straight and weak cowl reflection shock. The minimal total pressure loss, the lowest cowl drag, maximum mass-capture and the minimal aeroheating are achieved for the hypersonic inlet. For the other blunting manners, the ramp shock cannot reflect evenly and produces more curved cowl reflection shock. The Type V shock interference pattern occurs for the Cross Section Cutting blunting Manner (CSCM) and the strongest cowl reflection shock gives rise to the largest flow loss and drag. The cowl-lip blunted by the other two blunting manners is subjected to the shock interference pattern that transits with an increase in the blunting radius. Accordingly, the peak heat flux does not fall monotonously with the blunting radius increasing. Moreover, the cowl-lip surface suffers from severe aerothermal load when the shear layer or the supersonic jet impinges on the wall.
\end{abstract}

hypersonic inlet, cowl-lip bluntness, flow characteristics, shock pattern, shock interference

PACS number(s): 47.40.Ki, 47.40.Nm, 47.85.Gj

Citation: $\quad$ Lu H B, Yue L J, Chang X Y. Flow characteristics of hypersonic inlets with different cowl-lip blunting methods. Sci China-Phys Mech Astron, 2014, 57: 741-752, doi: 10.1007/s11433-013-5285-0

\section{Introduction}

As a critical component of scramjets, the hypersonic inlet serves to capture and compress incoming air for processing by the engine. The hypersonic inlet performs the important task of ensuring that the combustion chamber is supplied with a sufficient mass flow of air at the conditions required for supersonic combustion. To enable this task, a sharp leading edge should be theoretically used [1]. At hypersonic flight conditions (generally considered to be flight at Mach

*Corresponding author (email: yuelj@imech.ac.cn) number $>5$ ), the hypersonic inlets suffer from serious aeroheating problems. The severe aeroheating causes the sharp leading edge on the surfaces such as the forebody and cowl-lip, to be easily burned [2,3]. Additionally, a truly sharp leading edge would be not possible to manufacture. Hence, these surfaces have to be blunted to obtain acceptable heating levels.

Unfortunately, the blunted leading edges have an important influence on the wave-patterns, mass-capture, totalpressure recovery and cowl-drag of the hypersonic inlets. The forebody blunted leading edge produces a curved bow shock that generates a thick entropy layer. Under the strong entropy gradient, the subsonic airflow with a high tempera- 
ture and high pressure expands and accelerates to the supersonic range through the convergent-divergent streamtube, which forms between the bow shock and geometric surface. Thus, the forebody nose bluntness creates a significant change in the flowfield, such as shock positioning and air-capture characteristics, and adds complexity and difficulty to the design of the forebody surface $[1,4,5]$.

Compared with the forebody nose bluntness, the problem regarding the cowl-lip bluntness is more complex. In addition to the flow characteristics being similar to the forebody nose bluntness, the shock interference occurs when the external compression oblique shocks intersect the cowl bow shock. These phenomena degrade the performance of the hypersonic inlets, and complicate the internal flowfield. Shock-on-shock interaction is one of the most challenging research problems of gas dynamics and has been widely studied since the 1960s. Edney [6] formulated a detailed study of the entire spectrum of interference patterns and defined six types of shock interference patterns (Type I-VI). The results showed that the types of shock interference pattern and shock interference heating depended on the intersecting location of the impinging oblique shock relative to the curved bow shock. These shock interference patterns can cause high localized pressure and heat transfer rates on the surface, which can alter the aerodynamic characteristics of the vehicle and even lead to catastrophic failures [7-9]. Experimental and numerical investigations by Keyes et al. [10,11], Wieting et al. [12] and Yee et al. [13] showed the effects of gas thermodynamic properties, Reynolds number, Mach number and incident shock strength on the peak pressure and heat transfer rate. Borovoy et al. [14] further examined the influence of the location of the impinging shock on the surface heat transfer, focusing on the study of Type III and IV interactions. Analytical methods were also developed to predict the pressure and heat transfer resulting from shock interaction [15-17]. The above researches suggest that Type IV interference produces the most serious pressure and heat transfer. This interaction characterized by a supersonic jet embedded in the subsonic flow occurs when the incident oblique shock intersects the normal portion of the curved bow shock. The jet impingement leads to an extremely high pressure and heat transfer rate gradients over the narrow impingement region, and drastically deteriorates the aerothermal environment of the cowl leading edge of the hypersonic inlets. Subsequently, several researchers investigated how to control the Type IV shock interference pattern and decrease thermal loads [18-25]. These methods included the dispersed multiple shock-waves or isentropic compression waves configuration [18-21], opposed jet $[22,23]$ and pulsed laser energy deposition [24,25] in front of the cowl leading edge.

However, previous research mainly focused on the local shock interference patterns and did not take into account the effects on the flow characteristics and performance of the hypersonic inlets. The different cow-lip blunting methods can give rise to a significant change in shock patterns near the cowl-lip, as well as the flowfield, heat transfer and performance of the hypersonic inlets. Thus far, few studies have established a cowl-lip blunting method that can achieve good aerodynamic performance of the hypersonic inlets. How complex flow phenomena caused by shock interferences near the cowl-lip affect the performance of the hypersonic inlets is of research interest. The designing the blunting method has been considered to negate these negative effects. Based on the numerical results of a typical operating condition, this paper discusses the effect of different cowl-lip blunting methods on the flowfield and performance of the hypersonic inlets and provides a database for the cowl-lip blunting designs.

\section{Blunting methods}

To analyze the effects of the cowl-lip blunting methods on the flow characteristics and overall performance of the hypersonic inlets, four different blunting techniques are proposed, as shown in Figure 1. The dashed lines demonstrate the theoretical sharp cowl-lip. IS, CRS, UBS denote impinging shock, cowl reflection shock and undisturbed bow shock, respectively. For efficient operation at the design condition, the external compression waves intersect the theoretical sharp cowl-lip of the hypersonic inlets, which minimizes the spillage of compressed air.

ELM is a method usually used to blunt the forebody leading edge of the waveriders [26]. In the ELM, the cowl length of the hypersonic inlet with the blunted cowl-lip is identical to that of the hypersonic inlet with the theoretical sharp cowl-lip. The rounded cowl-lip surface is tangent to the inner geometric line of the inlet cowl, and the outer
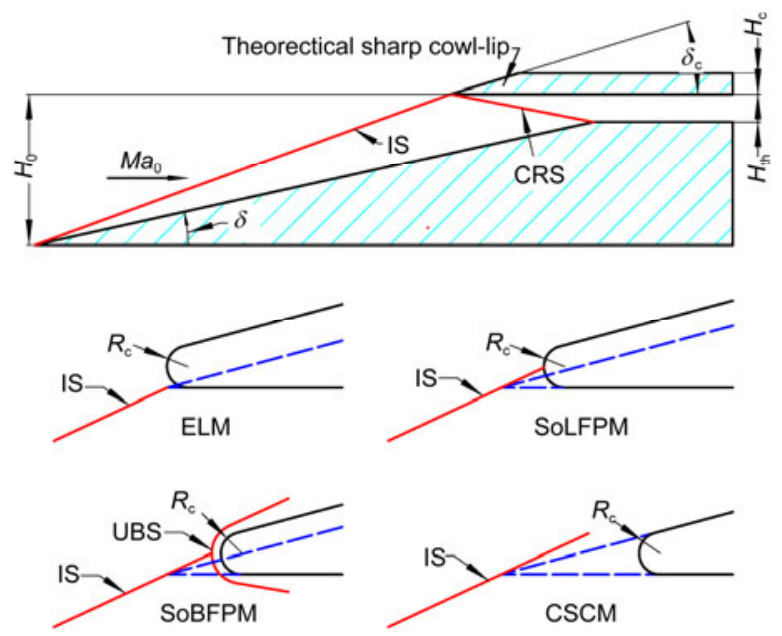

Figure 1 (Color online) Sketch of inlet model and blunting methods, where IS denotes impinging shock and UBS is undisturbed bow shock. 
geometric line relies on the blunting radius. Shock on Lip Frontal Point blunting Manner (SoLFPM) denotes that the incident ramp shock impinges on the frontal point of the blunted cowl-lip. The blunted circle is over the frontal point and tangent to the inner geometric line of the inlet cowl. Shock on undisturbed Bow shock Frontal Point blunting Manner (SoBFPM) represents that the incident ramp shock impinges on the vertex of the undisturbed cowl bow shock. The rounded surface tangent to the inner geometric line of the inlet cowl is determined by the standoff distance of the undisturbed cowl bow shock which can be obtained using the empirical relationship [27]. CSCM is a method which is generally used to blunt the forebody nose of the waveriders when the radius is much smaller than the vehicle size [28]. The blunting curve is an internally tangent circle whose center is over the internal bisector of the original sharp cowl-lip.

At the same radius, different cowl-lip blunting methods would yield different locations of the blunted cowl-lip. The cowl-lip locates downstream in sequence for the ELM, SoLFPM, SoBFPM and CSCM. Apparently, different cowl-lip blunting methods may lead to different intersecting locations of the impinging oblique shock relative to the curved bow shock. Effectively, the four different blunting methods feature different interference patterns caused by the external compression shock intersecting the cowl-lip bow shock. The associated flow phenomena represent the typical flow patterns of hypersonic inlet with a blunted cowl-lip at varied flight conditions. This study is helpful for a better understanding of flow mechanisms of hypersonic inlet with blunted cowl-lips and for optimization of the cowl-lip design.

\section{Inlet model}

A generic 2-D mixed compression inlet is employed to investigate the effects of the different cowl-lip blunting methods on the flowfield and performance of the hypersonic inlets, as shown in Figure 1. To highlight the key flow characteristics near the blunted cowl-lip and subsequent effects, the external compression is simplified as a single oblique shock. The corresponding flow deflection angle, $\delta$, is defined as $12.5^{\circ}$, which is equal to the total turning angle of the three ramps [29]. Under the design conditions of Mach number of 6 and flight altitude of $26 \mathrm{~km}$, the inlet with a theoretical sharp cowl-lip satisfies the following conditions: the external compression shock wave terminates with the apex of the sharp cowl-lip, and the cowl reflection shock is canceled at the ramp shoulder. The total contraction ratio of the inlet is 5.12. The inlet throat height, $H_{\mathrm{th}}$, is specified as $20 \mathrm{~mm}$. The isolator length is equal to five times the inlet throat height. A horizontal cowl is assumed with a geometric shape angle $\left(\delta_{\mathrm{c}}\right)$ of $17^{\circ}$ and a thickness $\left(H_{\mathrm{c}}\right)$ of $15 \mathrm{~mm}$.

\section{Numerical methods and validation}

\subsection{Numerical methods}

The validity of the governing equations and boundary conditions is a prerequisite for warranting the credibility of a numerical result. The radius of a blunted cowl-lip of the hypersonic inlets is usually small and approximately $1-3 \mathrm{~mm}$ [30], so the applicability of the flow equations and boundary conditions near the blunted cowl-lip need to be examined. The similarity parameter that governs this regime is Knudsen number, defined by $K n=\lambda / R_{\mathrm{c}}$, where $R_{\mathrm{c}}$ is the radius of the blunted cowl-lip; and $\lambda$ is the molecular mean free path. For convenience, $K n$ can be derived as follows [31]:

$$
K n=\frac{M a}{R e_{R_{\mathrm{c}}}} \sqrt{1.57 \gamma}=\frac{\mu}{\rho R_{\mathrm{c}}} \sqrt{\frac{1.57}{R_{\mathrm{g}} T}},
$$

here $M a, \gamma, R e_{R_{\mathrm{c}}}, \mu, \rho, T$ and $R_{\mathrm{g}}$ denotes Mach number, specific heat ratio, Reynolds number based on $R_{\mathrm{c}}$, viscosity, density, temperature and gas constant, respectively.

Figure 2 plots the variation of Knudsen number with the flight altitude $(h)$ at a constant blunting radius. The figure illustrates that $K n$ is lower than 0.03 when $R_{\mathrm{c}}$ is not less than $0.5 \mathrm{~mm}$ and the altitude is lower than $36 \mathrm{~km}$. This observation implies that the continuum Navier-Stokes equations and No-Slip boundary conditions are applicable to describe the flow regime near the blunted cowl-lip.

The full Navier-Stokes equations are numerically solved with the commercial software CFD++, which can efficiently resolve hypersonic flows and aerodynamic heating. The convection terms of the governing equations are discretized with a second-order total variation diminishing (TVD) method based on a new multi-dimensional interpolation framework. An approximate Riemann solver (HLLC) is used to define interface fluxes based on local wave-model solutions. The minmod limiter is employed to suppress spurious oscillations near the discontinuities while retaining high-order accuracy away from the jumps. The time terms

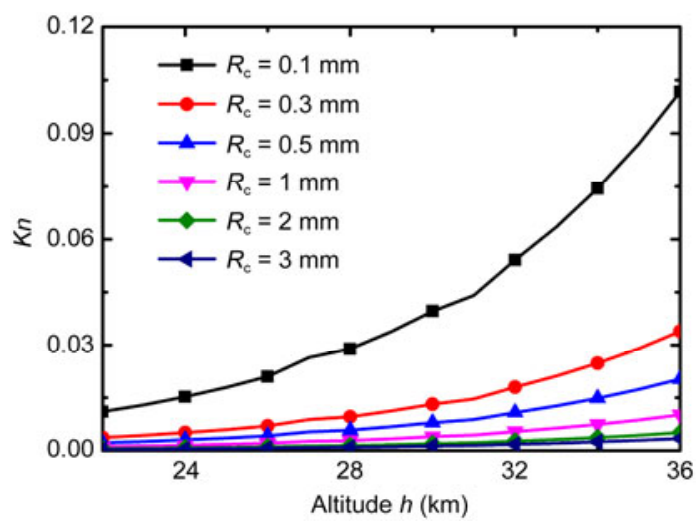

Figure 2 (Color online) Knudsen number versus the altitude and the blunted cowl-lip radius. 
are discretized with a second-order fully implicit scheme. The model of $k-\omega$ SST is used as the turbulence model with a compressibility correction for high Mach number flow. In addition, the multi-grid and dual time-step methods are employed to accelerate convergence.

\subsection{Code validation}

The 2-D mixed compression inlet $[5,32]$ is used to validate the reliability of the solver code. The mixed external/ internal compression inlet with a flat, horizontal cowl contains a forebody, which turns the flow outward using a combination of a blunted wedge and isentropic turning. The forebody is characterized by a nose bluntness with a radius of $0.127 \mathrm{~mm}$, initial wedge angle of $5^{\circ}$, and final ramp angle of $10^{\circ}$. The simulation conditions are listed in Table 1 [5]. The test gas is nitrogen. The first normal mesh spacing at the wall, $\Delta n$, is $1 \mu \mathrm{m} . y^{+}$at the forebody surface is less than 1, as shown in Figure 3. A comparison between the numerical and experimental results of the inner body surface pressure and heat transfer distributions is presented in Figure 4, where the heat transfer is expressed by the Stanton number, $S_{\mathrm{t}}=Q_{\mathrm{w}} /\left(1 / 2 \rho_{0} U_{0}^{3}\right)$. Figure 4 indicates that the numerical and experimental results are in good agreement, which shows that the present numerical technique can

Table 1 Test conditions given in ref. [5]

\begin{tabular}{ccccc}
\hline$M a_{0}$ & $R e / m$ & $P_{0}(\mathrm{~Pa})$ & $T_{0}(\mathrm{~K})$ & $T_{\mathrm{w}}(\mathrm{K})$ \\
\hline 10.39 & $1.87 \times 10^{7}$ & 606.74 & 48.72 & 294.44 \\
\hline
\end{tabular}

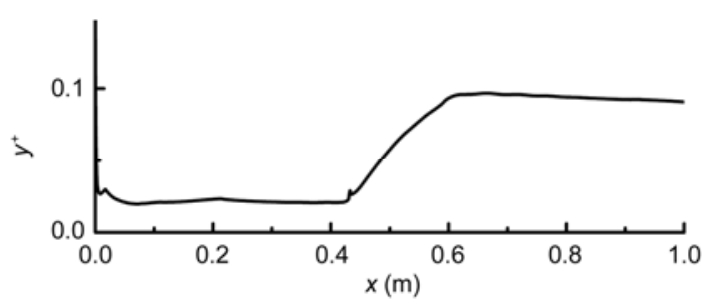

Figure $3 y^{+}$distribution at the inner body surface.
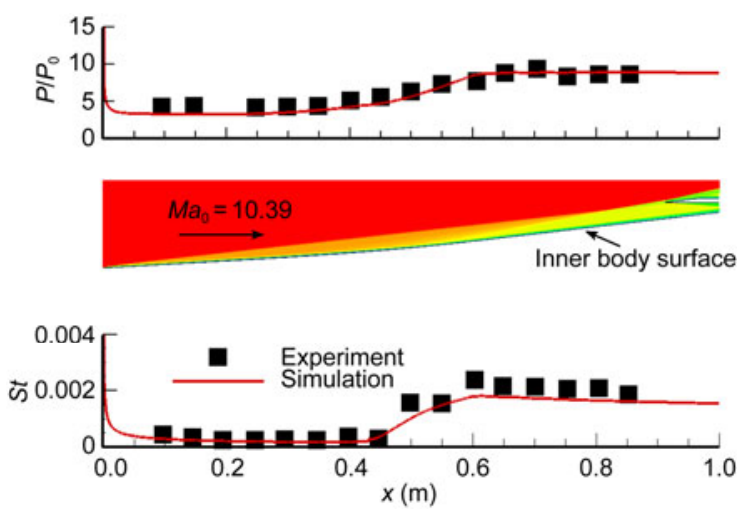

Figure 4 (Color online) Comparison of calculated and measured inner body surface pressure and Stanton number distributions. effectively capture the flow patterns and heat transfer at the wall.

\section{Results and discussion}

The current study discusses the effects of the four cowl-lip blunting methods on the internal flowfield and performance of the hypersonic inlets, under the design conditions of a flight Mach number of 6 and altitude of $26 \mathrm{~km}$. The simulations are conducted under the isothermal-constant temperature wall condition of $T_{\mathrm{w}}=294.44 \mathrm{~K}$. Through a survey of the effects of grid-refinement and the first mesh size normal to the wall, the computations are ultimately performed using about the total number of 0.23 million grids and the first mesh size of $1 \mu \mathrm{m}$.

\subsection{Flowfield and performance of the hypersonic inlet with a theoretical sharp cowl-lip}

To build a substantial base on studying the effects of cowllip blunting methods, this section briefly presents the flowfield and performance of the hypersonic inlet with a theoretical sharp cowl-lip. Figure 5 illustrates the pressure contour and streamlines. It can be found that the characteristics of the shock-on-lip, shock-on-shoulder and shock cancellation inside the inlet are nearly satisfied. The airflow decelerates to Ma3.2 across the straight cowl reflection shock. A small separation occurs upstream of the shoulder and induces a separation shock. However, the separation bubble is so small that the separation shock and reattachment shock cannot be clearly identified. The performance parameters, including mass-capture $(\varphi)$, total-pressure recovery $(\sigma)$, static-pressure-ratio $(\pi)$ and cowl-drag $(D)$, are listed in Table 2, where cowl drag is the streamwise integration of

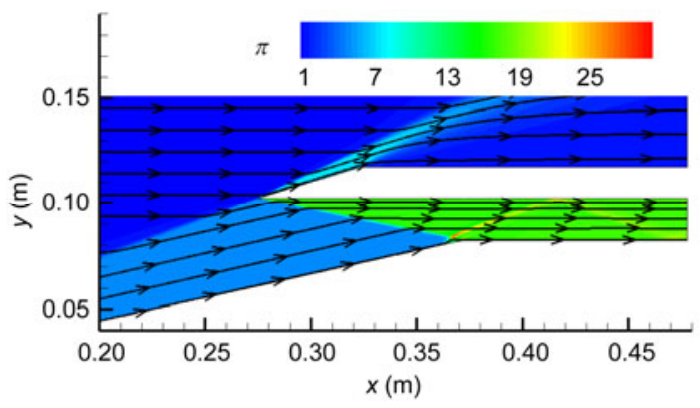

Figure 5 (Color online) Pressure contour and streamlines of the hypersonic inlet with a theoretical sharp cowl-lip.

Table 2 Performance parameters of the hypersonic inlet with a theoretical sharp cowl-lip

\begin{tabular}{cccc}
\hline $\begin{array}{c}\text { Mass-capture } \\
\text { coefficient }(\varphi)\end{array}$ & $\begin{array}{c}\text { Total-pressure } \\
\text { recovery }(\sigma)\end{array}$ & $\begin{array}{c}\text { Pressure } \\
\text { ratio }(\pi)\end{array}$ & $\begin{array}{c}\text { Cowl drag } \\
\left(\mathrm{D} / \mathrm{N} \cdot \mathrm{m}^{-1}\right)\end{array}$ \\
\hline 0.9997 & 0.4563 & 17.74 & 447.31 \\
\hline
\end{tabular}


the pressure and friction on the cowl wall per unit spanwise length. The inlet total pressure recovery is only $45.63 \%$, which is attributed to the large loss across one simplified strong external shock. However, the inlet total pressure recovery has no negative influence on our findings because our research lies in the relative effects of the cowl-lip blunting methods on the performance of the hypersonic inlets.

\subsection{Flow characteristics of the hypersonic inlets with blunted cowl-lip}

The flow characteristics of the hypersonic inlets with the cowl-lip blunted by the ELM, SoLFPM, SoBFPM and CSCM are analyzed in this section with the blunting radius of $2 \mathrm{~mm}$, whose ratio is 0.1 to the inlet throat height $\left(R_{\mathrm{c}} / H_{\mathrm{th}}\right.$ $=0.1$ ).

Figure 6 plots the Mach contours and streamlines near the blunted cowl-lip of the hypersonic inlets with the four blunting methods. The shock structures differ greatly from each other. From Figure 6(a), the cowl-lip blunted by ELM encounters the freestream. The incident ramp shock intersects below the lower sonic line of the undisturbed cowl bow shock. The strength of the ramp shock is much weaker than that of the cowl bow shock, hence, a $\lambda$ type of shock structure and shear layer is formed. The incident ramp shock and cowl bow shock merge into the right leg of the $\lambda$ shock. This is known as cowl reflection shock. The shear layer does not impinge on the cowl surface and enters into the inlet. The inlet internal flow downstream the cowl reflection shock is then divided into two regions by the shear layer. The first region is the core flow between the cowl reflection shock and the shear layer. The second region is the small amount of low-energy flow between the shear layer and the cowl wall, where the subsonic airflow with high pressure and temperature, originating from the stagnation region, expands and accelerates to low supersonic speed. Figure 7(a) further demonstrates the flow feature by plotting the profiles of Mach number at the plane $2 R_{\mathrm{c}}$ downstream of the blunted cowl-lip for the four different blunting methods. The definition of $Y_{\mathrm{cw}}$ is also included. The solid line corresponding to ELM shows two aforementioned regions above the cowl reflection shock where the air speed is Ma1.25 $\left(Y_{\mathrm{cw}} / R_{\mathrm{c}}=0-0.5\right)$ and Ma2.5 $\left(Y_{\mathrm{cw}} / R_{\mathrm{c}}=0.9-\right.$ $1.5)$, respectively. The airflow above the shear layer is of low total pressure because of the normal shock loss as shown in Figure 7(b). However, the length scale of the low energy (speed) flow path is very small, being only half of
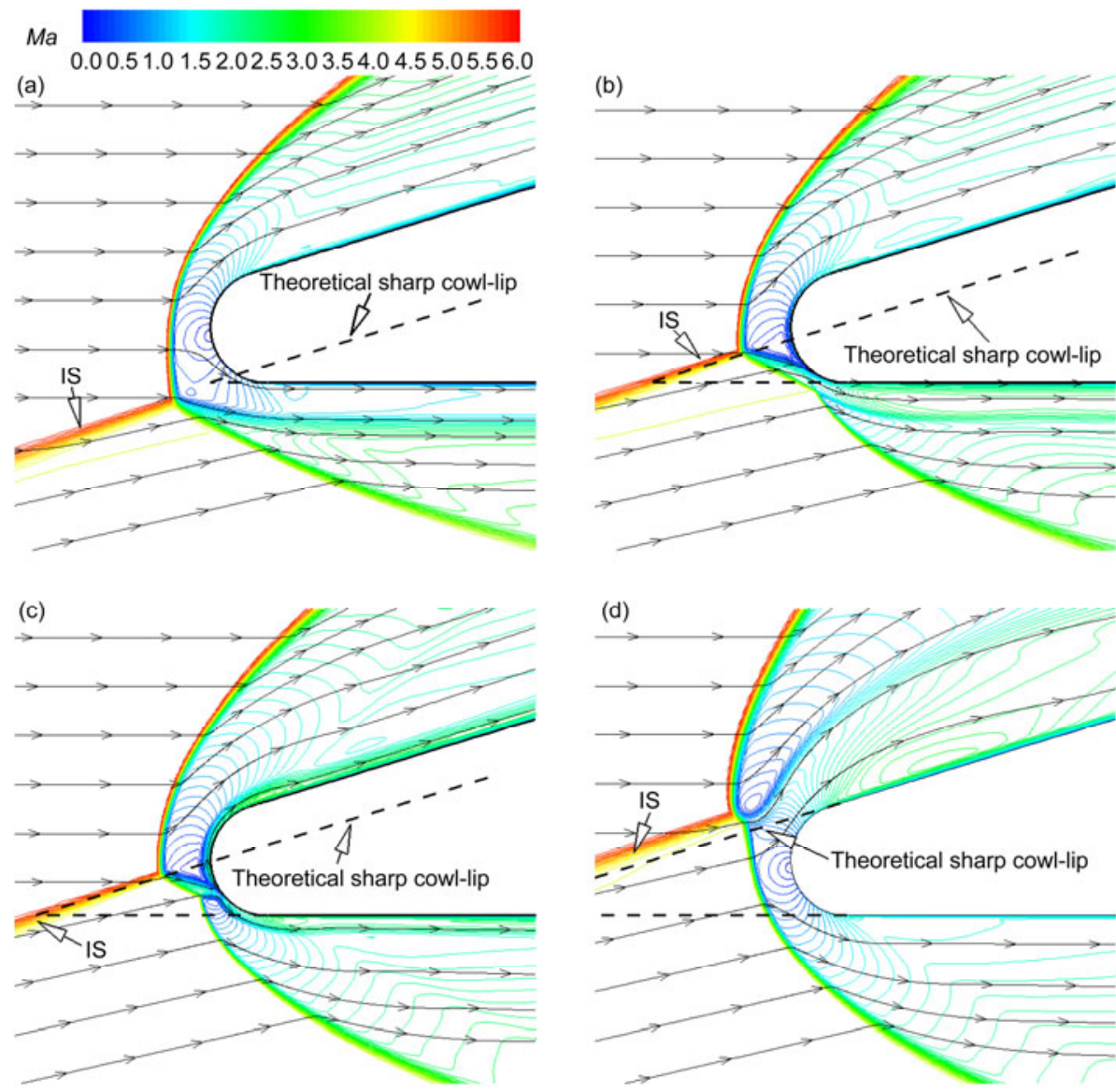

Figure 6 (Color online) Mach contours and streamlines near the blunted cowl-lip of the hypersonic inlets, where $R_{\mathrm{c}} / H_{\mathrm{th}}=0.1$. (a) ELM; (b) SoLFPM; (c) SoBFPM; (d) CSCM. 

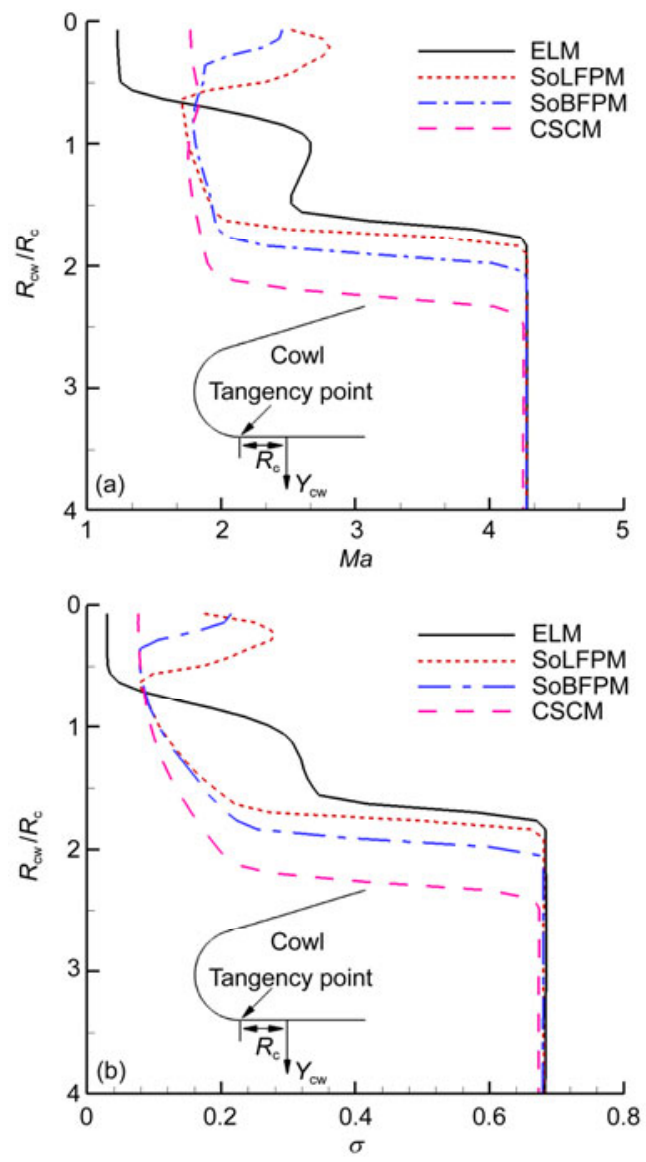

Figure 7 (Color online) Profiles of Mach number and total pressure recovery at the plane $2 R_{\mathrm{c}}$ downstream of the blunted cowl-lip for four different blunting methods. $Y_{\mathrm{cw}}$ is defined in the attached picture, which is 0 at the cowl wall. (a) Mach number profile; (b) total pressure recovery profile.

the blunting radius. This reveals that the airflow with low speed would not degrade the inlet performance substantially. It is not clear whether the shear layer swallowed by the inlet will affect the inlet buzz.

From Figure 6(b), the cowl-lip blunted by the SoLFPM is subjected to the Type III shock interference pattern, as defined by Edney [6]. The incident ramp shock intersects around the lower sonic line of the undisturbed cowl bow shock, which produces a transmitted shock and shear layer. The shear layer emanating from the shock intersection point attaches to the cowl surface with the subsonic flow above the layer turning upward and the supersonic flow below the layer passing through an oblique shock (the layer reflection shock) to turn parallel with the cowl wall surface. The interaction between the transmitted shock and the layer reflection shock produces a curved cowl reflection shock and shear layer. The shear layer is also in close proximity to the wall and separates two regions. Figure 7(a) further shows that the airflow above the shear layer is more rapid because it goes across three oblique shock waves, namely, the incident ramp shock, the transmitted shock and the layer reflection shock, rather than a normal shock for the ELM shown in Figure 6(a). Higher total pressure ratio is thus achieved (see Figure 7(b)). In the other region below the shear layer, the airflow with the SoLFPM is much slower than that with the ELM because the cowl reflection shock becomes more curved (larger shock angle). Correspondingly, larger total pressure loss can be observed for the SoLFPM.

From Figure 6(c), the cowl-lip blunted by the SoBFPM is subjected to the Type IV shock interference pattern, as defined by Edney [6]. The incident ramp shock intersecting the normal portion of the cowl bow shock produces a supersonic jet embedded in the subsonic flow. The jet impinges on the cowl surface and ends up with a normal shock. Through the terminal shock, the flow in the supersonic jet bounded by the shear layers is divided into two parts, and passes outside and inside the inlet respectively. A thin supersonic flow arising from the terminal shock exists near the cowl wall, as shown in Figures 6(c) and 7(a). The speed is lower than that with the SoLFPM because of more complex shocks and larger pressure loss in the supersonic jet. Below this region, the airflow is affected primarily by the curved cowl reflection shock. Similar to those with the ELM and SoLFPM, the reflection shock becomes weaker as it is farther away from the wall, contributing to the gradually increased airflow speed and reduced total pressure loss, as shown in Figure 7(b).

From Figure 6(d), the cowl-lip blunted by the CSCM encounters the airflow behind the ramp shock. The ramp shock intersects above the upper sonic line of the cowl bow shock, resulting in the Type $\mathrm{V}$ shock interference pattern. A triple shock type and a shear layer occur. The shear layer is not swallowed by the inlet and large spillage exists. Unlike that in other blunting methods, near-wall region characterized by different air speed does not occur.

Figure 8 further illustrates the internal flowfield of the hypersonic inlets with the cowl-lip blunted by different methods. The streamwise position of the cowl-lip frontal point is labeled, together with the separation and reattachment points of the separation bubble. Compared with Figure 5 , it is found that the cowl-lip blunting techniques have a substantial impact on the internal flowfield of the hypersonic inlets. The cowl reflection shock impinges upstream of the shoulder for the four blunting methods due to the larger cowl reflection shock angle than that for theoretical sharp cowl-lip. Higher adverse pressure gradient then contributes to a larger separation bubble. The separation shock and the reattachment shock can be distinctly identified and reflect back and forth in the internal flow path. The interaction of the shoulder expansion fan, separation shock and reattachment shock adds complexity in the flowfield of the hypersonic inlets.

Furthermore, it can be seen that the further downstream the blunted cowl-lip is located, the further upstream the position is located, on which the cowl reflection shock impinges the ramp. For example, the cowl-lip blunted by the CSCM is positioned the farthest downstream, while the 

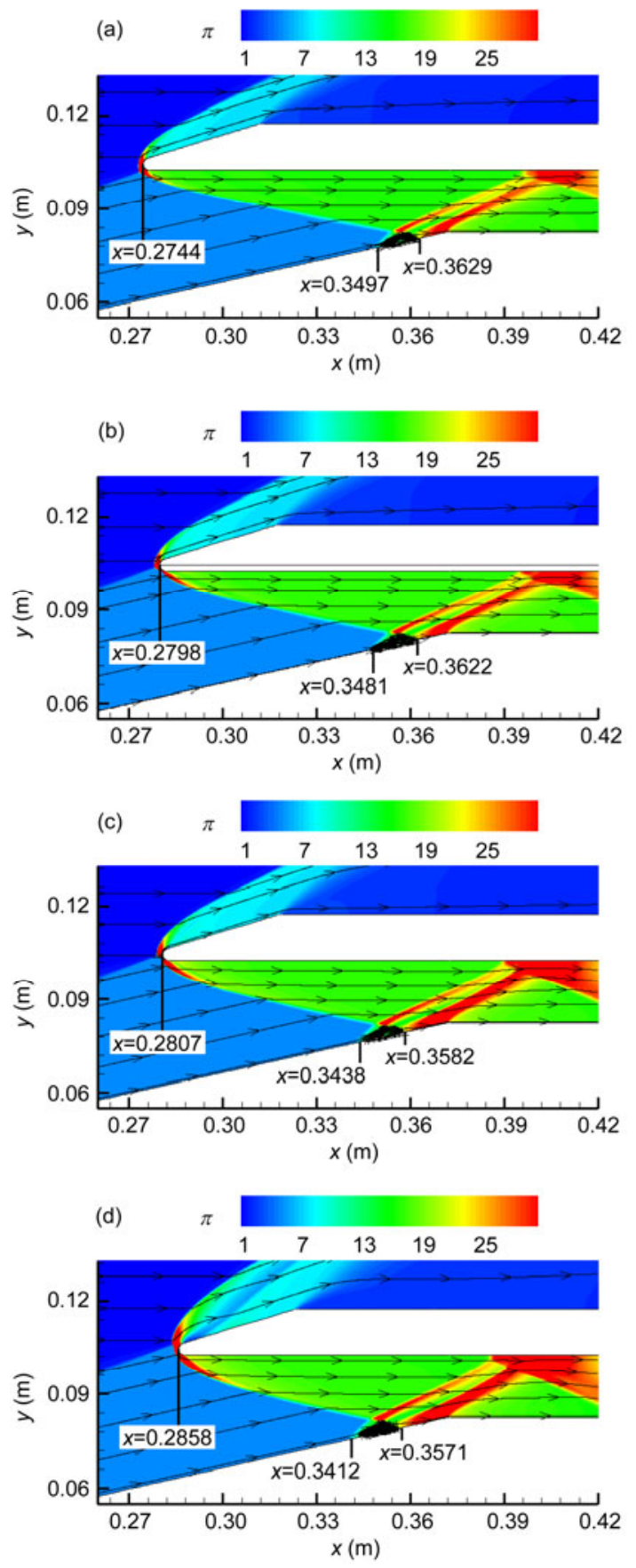

Figure 8 (Color online) Pressure contours and streamlines of hypersonic inlets with a blunted cowl-lip, where $R_{\mathrm{c}} / H_{\mathrm{th}}=0.1$. (a) ELM; (b) SoLFPM; (c) SoBFPM; (d) CSCM.

cowl reflection shock impinges the farthest upstream of the ramp. This phenomenon is attributed to the different shock structures near the blunted cowl-lip. Note in Figure 6, for the ELM, a nearly even reflection of the incident ramp shock produces an approximately straight cowl reflection shock, whose shock angle is the smallest. For the SoLFPM, SoBFPM and CSCM, the incident ramp shock cannot reflect evenly and creates a curved and non-equal strength cowl reflection shock because of the blocked effects of the blunted geometric surface shown in Figures 6(b)-(d). Figure 7 illustrates that location of the cowl reflection shocks moves downward for the blunting method of ELM, SoLFPM, SoBFPM and CSCM. This indicates the shock becomes steeper and stronger which results in larger separation.

Thus, different cowl-lip blunting methods trigger significant alterations in the cowl reflection shock structures and the associated flow patterns, even when the blunting radius is very small. This change will affect the hypersonic inlets performance and thermal loads.

\subsection{Thermal loads on the cowl-lips with different blunting methods}

The corresponding heat transfer distributions at the cowl-lip circular surface with the blunting radius of $2 \mathrm{~mm}$ are plotted against the circumferential angle $(\theta)$, in Figure 9. The heat transfer rates are normalized by the stagnation point heat flux at the freestream condition. The corresponding stagnation point heat flux, $Q_{\mathrm{st}}$, is $2.88 \mathrm{MW} / \mathrm{m}^{2}$. It should be noted that there is a large difference among the four heat flux distributions. Specially, the heat flux distribution for the SoBFPM presents an asymmetrical bell-curve shape, and a local large temperature gradient exists at the supersonic jet impingement. Additionally, the peak heat flux corresponding to the ELM, SoLFPM, SoBFPM and CSCM is 0.91, 1.61, 7.11 and 1.81 times of the stagnation point heat flux, respectively. These differences are attributed to the different shock structures near the blunted cowl-lip, as described in last section. The Type IV shock interference pattern for the SoBFPM contributes to extreme severe aeroheating.

The blunting radius can exert an important influence on the thermal loads on the cowl-lip. The peak heat flux on the blunted cowl-lip surface is plotted against the radius for the four blunting methods in Figure 10. The peak heat flux is normalized by the freestream kinetic energy, $1 / 2 \rho_{0} U_{0}^{3}$. It is

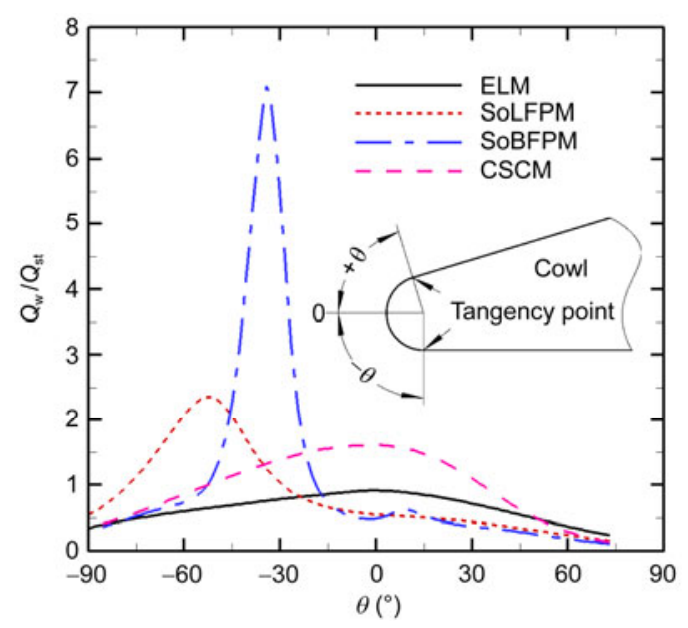

Figure 9 (Color online) Distributions of the cowl circular wall heat transfer for the four blunting methods, where $R_{\mathrm{c}} / H_{\mathrm{th}}=0.1$. 


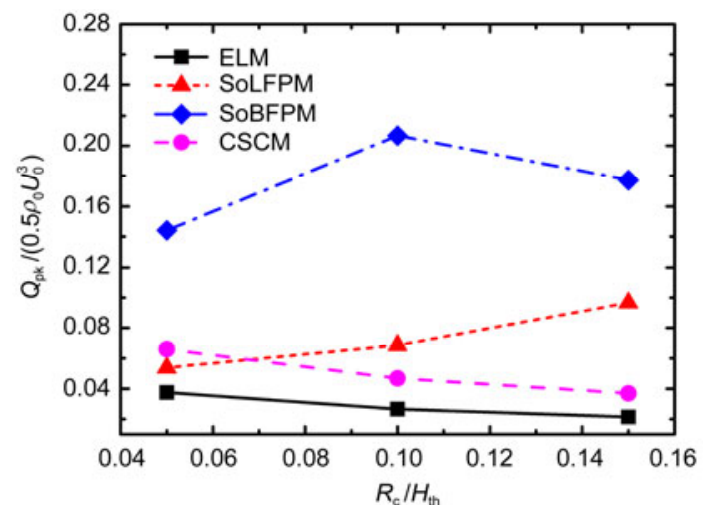

Figure 10 (Color online) Variation of the peak heat flux on the blunted curved cowl-lip surface with the blunting radius for the four blunting methods.

known that aeroheating can decrease with an increase in the radius. The peak heat flux for the ELM and CSCM exhibits a similar tendency. However, in Figure 10, a surprising result can be found in that the peak heat flux of the SoLFPM increases with an increase of the blunting radius, and the peak heat flux of the SoBFPM is a maximum at a certain radius. These results can be attributed to the change of shock structures with the blunting radius. For the SoLFPM and SoBFPM, the shock interference pattern dominated by the interaction of the shear layer with the cowl-lip is sensitive to the blunting radius. Combining Figure 6(b) $\left(R_{\mathrm{c}} / H_{\mathrm{th}}=0.1\right)$ with Figure 11 , it can be observed that when the blunting radius increases, the shock interference pattern turns from Type $\mathrm{II}^{+}$to Type III for the SoLFPM. In Type $\mathrm{II}^{+}$, the shear layer grasps the cowl surface, while in Type III, the shear layer impinges on the cowl surface, which will increase the peak heat flux. Combining Figure 6(c) $\left(R_{\mathrm{c}} / H_{\mathrm{th}}=0.1\right)$ with Figure 12 , it can be observed that the type of the interference pattern also varies with the blunting radius. A Type III interference pattern occurs at a small blunting radius, while a Type IV interference pattern results at a larger blunting radius $\left(R_{\mathrm{c}} / H_{\mathrm{th}}=0.1\right)$, leading to an increase of the peak heat flux with the blunting radius. With a further increase of the blunting radius, the type IV interference pattern holds, leading to a reduction of the peak heat flux with the blunting radius increasing.

\subsection{Effect of different cowl-lip blunting methods on the hypersonic inlets performance}

Total pressure recovery, pressure rise, mass-capture and cowl drag of the hypersonic inlets is investigated in this part. The radius of the blunted cowl-lip is specified as $1 \mathrm{~mm}$, $2 \mathrm{~mm}$ and $3 \mathrm{~mm}$. The corresponding ratio of the blunting radius to the inlet throat height is $0.05,0.1$ and 0.15 $\left(R_{\mathrm{c}} / H_{\mathrm{th}}=0.05,0.1,0.15\right)$.

\subsubsection{Effect on total pressure recovery and pressure rise}

The total pressure recovery and pressure rise at the isolator
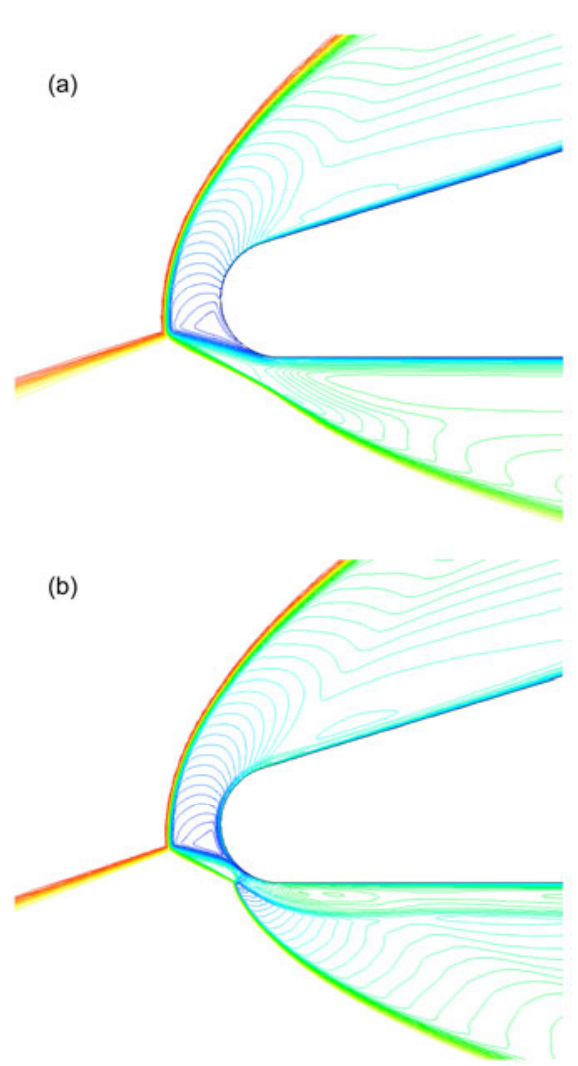

Figure 11 (Color online) Mach contours near the blunted cowl-lip with different radius for the SoLFPM. (a) $R_{\mathrm{c}} / H_{\mathrm{th}}=0.05$; (b) $R_{\mathrm{c}} / H_{\mathrm{th}}=0.15$.

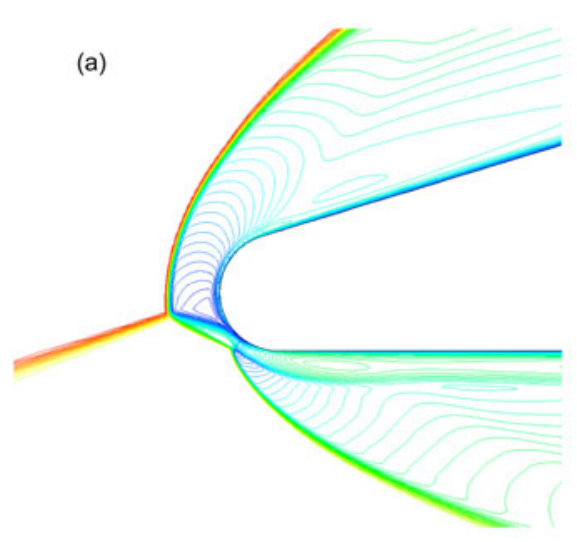

(b)

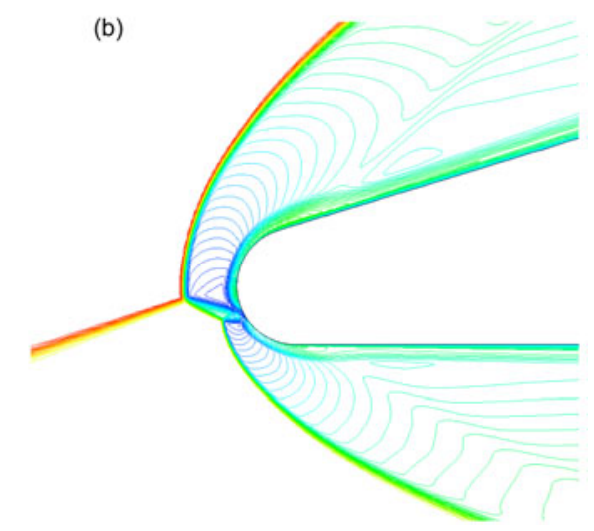

Figure 12 (Color online) Mach contours near the blunted cowl-lip with different radius for the SoBFPM. (a) $R_{\mathrm{c}} / H_{\mathrm{th}}=0.05$; (b) $R_{\mathrm{c}} / H_{\mathrm{th}}=0.15$. 
exit are plotted against the blunting radius for the four blunting methods in Figure 13. The parameters are normalized by those of the inlet with a theoretical sharp cowl-lip. The horizontal axis is the ratio of the blunting radius to the inlet throat height. It can be noted from Figure 13(a) that the total pressure recovery with the blunted cowl-lip is lower than the pressure recovery with a theoretical sharp cowl-lip. Furthermore, the total pressure loss increases dramatically with an increase in the blunting radius. This result implies that the blunted leading edge degrades the total pressure recovery. On one hand, the shock interference alters the shape of the cowl reflection shock, which results in steeper shock and larger shock loss. For the sharp cowl-lip, the total pressure ratio to the incoming flow is $55 \%$ for the airflow downstream the straight cowl reflection shock. For the blunted cowl-lip, the pressure loss is much larger, particularly in the region not far from the wall, as plotted in Figure 7(b). On the other hand, the characteristics of the separation and reattachment shocks in the inlet internal flowfield also increase the total pressure loss, as shown in Figure 8.

At the same blunting radius, the inlet total pressure recovery with the ELM is significantly higher than that with the other three methods, particularly for the large blunting radius. The differences in the total pressure loss for the four blunting methods are caused by changes in the shape of the different cowl reflection shocks resulting from the different shock interferences near the blunted cowl-lip. As shown in Figure 7(b), the total pressure recovery profiles differ from each other for the four blunting methods. The ELM generates an approximately straight and the weakest cowl reflection shock because of the even reflection of the incident ramp shock (see Figure 6(a)). Although there is a low energy region above the shear layer, it does not degrade the hypersonic inlet performance significantly because the flow path is very small compared to the inlet throat. The pressure recovery becomes lower in sequence for the SoLFPM, SoBFPM and CSCM, because of the stronger curved cowl reflection shock. For the SoLFPM, a Type $\mathrm{II}^{+}$shock interference pattern occurs and generate a nearly straight cowl reflection shock similar to that for the ELM when the blunting radius is small $\left(R_{\mathrm{c}} / H_{\mathrm{th}}=0.05\right)$. With an increase in the blunting radius, a Type III shock interference pattern occurs, and the attached shear layer contributes to stronger curved cowl reflection shock and larger total pressure loss. For the SoBFPM, a Type IV shock interference pattern strengthens the cowl reflection shock. For the CSCM, the curved cowl reflection shock is the strongest, because the blunted cowl-lip is fully submerged in the flow behind the incident ramp shock.

Another feature that can be observed is that the inlet total pressure recovery with the cowl-lip blunted by the ELM, SoLFPM, SoBFPM and CSCM drops by $3.6 \%, 3.8 \%, 9.8 \%$ and $15.0 \%$, respectively, compared to the pressure recovery with the theoretical sharp cowl-lip, when $R_{\mathrm{c}} / H_{\mathrm{th}}=0.05$. This data implies that the cowl-lip blunting methods have a sig- nificant impact on the total pressure recovery even though the blunting radius is small.

It can be observed from Figure 13(b) that the pressure rise of the inlet with the blunted cowl-lip departures pronouncedly from the designed value. The pressure rise with the blunted cowl-lip is higher than the one with a theoretical sharp cowl-lip and increases dramatically with an increase in the blunting radius. The tendency of pressure rise is contrary to the trend of the total pressure recovery for the four blunting methods because of approximately constant inlet contraction ratio. The pressure rise with the ELM is the most consistent to the inlet design condition.

\subsubsection{Effect on mass-capture}

The mass flow rate captured by the hypersonic inlets with the cowl-lip blunted by four methods is plotted against the radius in Figure 14. The mass flow rate is normalized by the flow rate that is captured by the inlet with a theoretical sharp cowl-lip. The large differences in the inlet masscapture can be observed among the four blunting methods. The inlet captured flow rate with the ELM, SoLFPM and SoBFPM is higher than the flow rate captured by the inlet with a theoretical sharp cowl-lip. The rate rises linearly with an increase in the blunting radius. The inlet captured flow rate with the CSCM is lower than the flow rate captured by the inlet with a theoretical sharp cowl-lip, and the rate reduces linearly with an increase in the blunting radius.
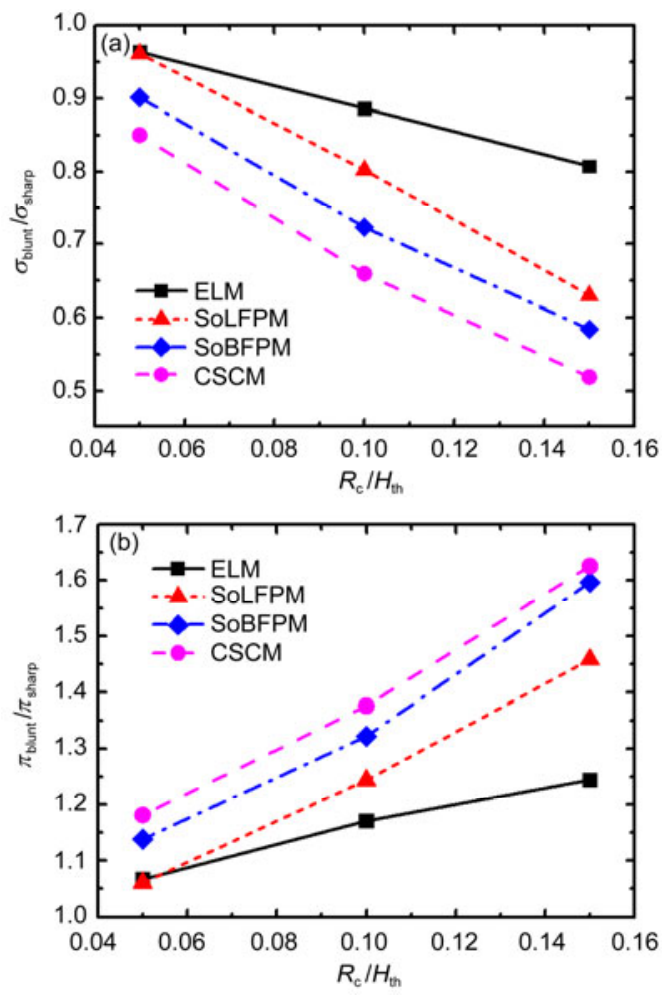

Figure 13 (Color online) Variation of flow parameters at the isolator exit with the blunting radius for the four blunting methods. (a) Total pressure recovery; (b) pressure rise. 


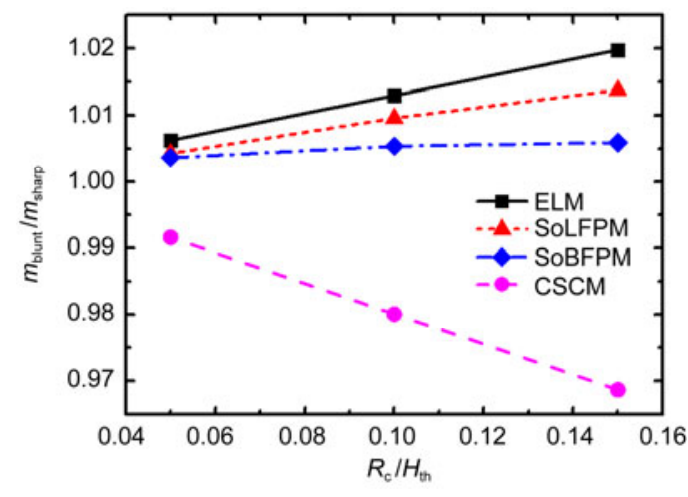

Figure 14 (Color online) Variation of the mass flow rate captured by the hypersonic inlets with the blunting radius for the four blunting methods.

According to Figures 6 and 8, these differences can be attributed to two key factors. First, the increased projected frontal area caused by the cowl-lip bluntness provides a potential extra mass flow. Second, as plotted in Figure 6, the cowl-lip position locating downstream in sequence for the ELM, SoLFPM, SoBFPM and CSCM results in a gradually increased spillage and a reduced mass-capture under the same blunting radius. For the CSCM, a large spillage occurs because the theoretical sharp cowl-lip is directly cut backward and the cowl-lip position lies far downstream.

\subsubsection{Effect on cowl drag}

The cowl drag is plotted against the blunting radius for the four blunting methods in Figure 15. The vertical axis denotes the cowl-drag ratio of the hypersonic inlets with a blunted cowl-lip to that with the sharp cowl-lip. The cowl drag is noticeably increased by the cowl-lip bluntness and increases drastically with an increase in the blunting radius. In particular, the inlet cowl drag from the ELM is far less than that from the other blunting methods. As defined previously, the cowl drag consists of pressure and friction. However, the cowl drag increment is primarily derived from the streamwise integration of the cowl surface pressure, rather than friction. Figure 16 shows the pressure distributions on the cowl surface. It can be determined that the pressure differences originate from the curved surface (BC segment), which is produced by the shock interaction.

Based on the report of Tang and Chase [33], the specific impulse of the hydrocarbon-fueled scramjet can be taken as approximately $800 \mathrm{~s}$ at the stoichiometric equivalence ratio. According to the mass flow rate captured by the hypersonic inlet with a theoretical sharp cowl-lip, the percentage of the cowl drag increment to the engine thrust can be calculated. At $R_{\mathrm{c}} / H_{\mathrm{th}}=0.05$, the percentage for the ELM, SoLFPM, SoBFPM and CSCM is $2.0 \%, 3.2 \%, 4.2 \%$ and $7.4 \%$, respectively. At $R_{\mathrm{c}} / H_{\mathrm{th}}=0.1$, the percentage for the ELM, SoLFPM, SoBFPM and CSCM is $5.1 \%, 8.8 \%, 10.9 \%$ and $17.0 \%$, respectively. These results indicate that the cowl-lip blunting method has a major influence on the aerodynamic force.

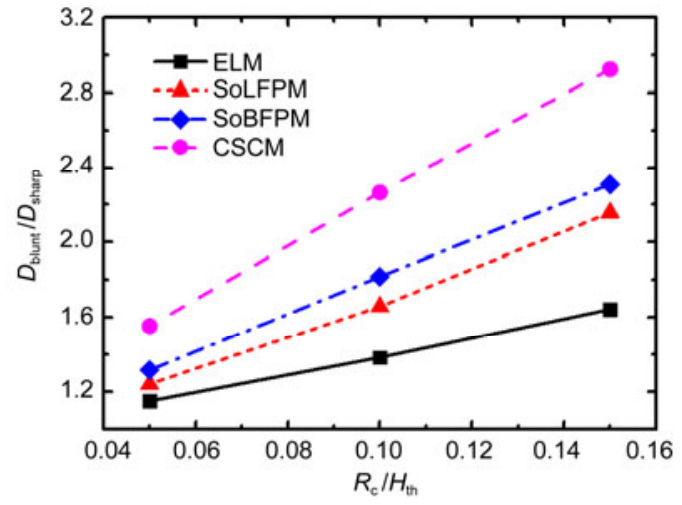

Figure 15 (Color online) Variation of the cowl drag with the blunting radius for the four blunting methods.

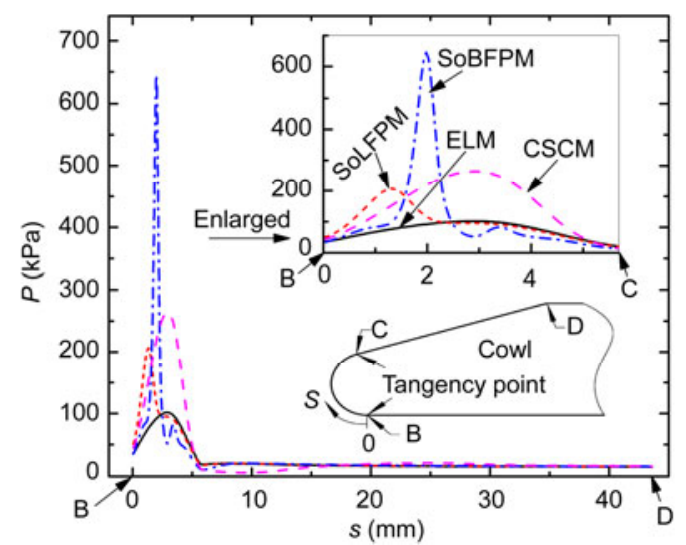

Figure 16 (Color online) Cowl wall pressure distribution for the four blunting methods, where $R_{\mathrm{c}} / H_{\mathrm{th}}=0.1$.

As discussed above, the cowl-lip blunting methods have an important effect on the flowfield and performance of the hypersonic inlets. Among the four blunting methods proposed in this paper, the ELM produces the smallest change in the flow patterns of the hypersonic inlets, and the hypersonic inlet with the cowl-lip blunted by the ELM has the most optimal performance, including mass-capture, total pressure recovery, cowl drag and minimum heat loads. Consequently, the cowl-lip of the hypersonic inlet should be blunted by the ELM.

\section{Conclusions}

The sharp cowl-lip leading edge has to be blunted to obtain acceptable heating levels for hypersonic inlet. This paper proposes four blunting methods, the ELM, SoLFPM, SoBFPM and CSCM, and further investigates in detail the flow characteristics and performances of generic hypersonic inlets with the blunted cowl-lips by numerical simulation. The flow mechanism regarding the effect of the cowl-lip blunting methods is also elucidated.

The flowfields in the vicinity of the blunted cowl-lips for the different blunting methods feature the typical shock in- 
terference patterns caused by the external compression shock intersecting the cowl bow shock. These local shock interference patterns have a major influence on the shock structures in the hypersonic inlets even though the blunting radius is very small, which are responsible for the performance degradation of the hypersonic inlets. With an increase in the blunting radius, the total pressure recovery falls sharply, and the cowl drag rises rapidly. However, the mass-capture for the ELM, SoLFPM and SoBFPM increases slightly.

Among the four blunting methods, the performance of the hypersonic inlets with the cowl-lip blunted by the ELM is the most optimal. For the ELM, the incident ramp shock intersects below the sonic line of the cowl bow shock. A nearly even reflection gives rise to an approximately straight and weak cowl reflection shock, which produces the smallest change in the flow patterns of the hypersonic inlets. The minimal total pressure loss, the lowest cowl drag and the maximum mass-capture are thus achieved along with minimal aerothermal load. For the other blunting methods, the ramp shock cannot reflect evenly and produces a stronger curved cowl reflection shock. This shock structure dramatically deteriorates the hypersonic inlets performance. The CSCM produces the Type V shock interference pattern associated with a large spillage and the cowl-lip encountering the flow behind the ramp shock. The strongest bow cowl reflection shock occurs, resulting in the largest cowl drag and the lowest total pressure recovery. The cowl-lip blunted by the SoLFPM is subjected to the shock interference pattern that changes from Type $\mathrm{II}^{+}$to Type III with an increase in the blunting radius. For the SoBFPM, Type III or IV shock pattern occurs, coupled with more curved cowl reflection shock. The cowl-lip surface suffers from severe aerothermal load when the shear layer or the supersonic jet impinges on the wall. Unlike that with the ELM and CSCM, the peak heat flux of the SoLFPM rises with an increase in the blunting radius, and the peak heat flux of the SoBFPM is a maximum at a certain radius. These results can be attributed to the transition of shock interference pattern with the blunting radius.

In particular, a near-wall region characterized by different air speed occurs for the ELM, SoLFPM and SoBFPM, which has little effect on the inlet performance. This needs to be further studied whether the shear layer swallowed by the inlet will affect the inlet buzz.

The four blunting methods and associated flow phenomena represent the typical flow patterns of hypersonic inlet with blunted cowl-lip at varied flight conditions. This research elucidates the flow mechanism and would aid in understanding the flow and performance of the hypersonic inlets with blunted cowl-lip.

This work was supported by the National Natural Science Foundation of China (Grant Nos. 90716014 and 91216115). The authors wish to thank all the members of our work group.
1 Curran E T, Murthy S N B. Scramjet Propulsion. Reston, VA: AIAA, 2000. 447-511

2 Bertin J J. Hypersonic Aerothermodynamics. Washington, DC: AIAA, 1994. 240-267

3 Anderson J D. Hypersonic and High Temperature Gas Dynamics. 2nd ed. Reston, VA: AIAA, 2000. 346-355

4 Sanator R J, Boccio J L, Shamshins D. Effect of Bluntness on Hypersonic Two-dimensional Inlet Type Flows. Technical Report, NASA CR-1145, Washington, DC. 1968

5 Ault D, Van Wie D. Experimental and computational results for the external flowfield of a scramjet inlet. J Propul Power, 1994, 10(4): 533-539

6 Edney B. Anomalous Heat Transfer and Pressure Distributions on Blunt Bodies at Hypersonic Speeds in the Presence of an Impinging Shock. Technical Report, FFA 115, Aeronautical Research Institute of Sweden. 1968

7 Ryan B. Summary of the Aerothermodynamic Interference Literature. Technical Report, TN 4051-160, Naval Weapons Center (China Lake, Calif.). 1969

8 Korkegi R H. Survey of viscous interactions associated with high Mach number flight. AIAA J, 1971, 9(5): 771-784

9 Watts J D. Flight Experience with Shock Impingement and Interference Heating on the X-15-2 Research Airplane. Technical Report, NASA TM X-1669, Washington, DC. 1968

10 Keyes J W, Hains F D. Analytical and Experimental Studies of Shock Interference Heating in Hypersonic Flows. Technical Report, NASA TN D-7139, Washington, DC. 1973

11 Morris D J, Keyes J W. Computer Programs for Predicting Supersonic and Hypersonic Interference Flow Fields and Heating. Technical Report, NASA TM X-2725, Washington, DC. 1973

12 Wieting A R, Holden M S. Experimental study of shock wave interference heating on a cylindrical leading edge at Mach 6 and 8. AIAA J, 1989, 27(11): 1557-1565

13 Yee H C, Klopfer G H, Montagne J L. High-resolution shock capturing schemes for inviscid and viscous hypersonic flows. J Comput Phys, 1990, 88(1): 31-61

14 Borovoy V, Chinilov A, Gusev V, et al. Interference between a cylindrical bow shock and a plane oblique shock. AIAA J, 1997, 35(11): 1721-1728

15 Frame M, Lewis M. Analytical solution of the type IV shock interaction. J Propul Power, 1997, 13(5): 601-609

16 Lind C, Lewis M. Computational analysis of the unsteady type IV shock interaction of blunt body flows. J Propul Power, 1996, 12(1): 127-133

17 Lind C. Effect of geometry on the unsteady type-IV shock interaction. J Aircraft, 1997, 34(1): 64-71

18 Wieting A R. Multiple shock-shock interference on a cylindrical leading edge. AIAA J, 1992, 30(8): 2073-2079

19 Hsu K, Parpia I H. Simulation of multiple shock-shock interference patterns on a cylindrical leading edge. AIAA J, 1996, 34(4): 764-771

20 Wang W, Xie L, Guo R. Influence of blunting manner of the lip highlight of hypersonic inlet on the aerothermodynamic performance. AIAA paper 2011-2306. 2011

21 Lu H B, Yue L J, Xiao Y B, et al. Interaction of isentropic compression waves with a bow shock. AIAA J, 2013, 15(10): 2474-2484

22 Prabhu R, Thareja R, Wieting A R. Computational studies of a fluid spike as a leading edge protection device for shock-shock interference heating. AIAA paper 91-1734. 1991

23 Albertson C W, Venkat V S. Shock Interaction control for scramjet cowl leading edges. AIAA paper 2005-3289. 2005

24 Kandala R, Candler G V. Numerical studies of laser-induced energy deposition for supersonic flow control. AIAA J, 2004, 42(11): 2266-2275

25 Adeleren R G, Hong Y, Elliott G S, et al. Control of Edney IV interaction by pulsed laser energy deposition. AIAA J, 2005, 43(2): 
256-269

26 Tincher D, Lane J. On the design of a hypersonic waverider test bed vehicle: A first step to outer planet exploration. AIAA Paper, AIAA92-0308. 1992

27 Billig F S. Shock-wave shapes around spherical and cylindrical-nosed bodies. J Spacecraft Rockets, 1967, 4(6): 822-823

28 Takashima N, Lewis M J. Navier-Stokes computations of a viscous optimized waverider. AIAA paper 92-0305. 1992

29 Van Wie D, Drewry D, King D, et al. The hypersonic environment: Required operating conditions and design challenges. J Mater Sci,
2004, 39(19): 5915-5924

30 Sha $\mathrm{P}$, Zhengyu $\mathrm{T}$, Dinghua $\mathrm{F}$, et al. Computation and analysis of aeroheating on hypersonic inlet leading edge. AIAA Paper, AIAA2009-7370. 2009

31 Shen C. Rarefied Gas Dynamics. New York: Springer Berlin Heidelberg, 2005. 13-14

32 Van Wie D, Ault D. Internal flowfield characteristics of a scramjet inlet at Mach 10. J Propul Power, 1996, 12(1): 158-164

33 Tang M, Chase $\mathrm{R}$ L. The quest for hypersonic flight with air-breathing Propulsion. AIAA Paper, AIAA-2008-2546. 2008 